\title{
Commutators and Anti-Commutators of Idempotents in Rings
}

\author{
Dinesh Khurana and T. Y. Lam
}

\begin{abstract}
We show that a ring $R$ has two idempotents $e, e^{\prime}$ with an invertible commutator $e e^{\prime}-e^{\prime} e$ if and only if $R \cong \mathbb{M}_{2}(S)$ for a ring $S$ in which 1 is a sum of two units. In this case, the "anti-commutator" $e e^{\prime}+e^{\prime} e$ is automatically invertible, so we study also the broader class of rings having such an invertible anti-commutator. Simple artinian rings $R$ (along with other related classes of matrix rings) with one of the above properties are completely determined. In this study, we also arrive at various new criteria for general $2 \times 2$ matrix rings. For instance, $R$ is such a matrix ring if and only if it has an invertible commutator $e r-r e$ where $e^{2}=e$.
\end{abstract}

\section{$\S 1$. Introduction}

The work in this paper was inspired by an insightful exercise of Kaplansky in his 1968 book [Ka] on rings of operators. Given two idempotents $e, e^{\prime}$ in a ring $R$ with the property that their commutator $e e^{\prime}-e^{\prime} e$ is invertible, "Exercise 6" in [Ka: p. 25] asked the readers to show that the idempotents $e, e^{\prime}, 1-e, 1-e^{\prime}$ are pairwise isomorphic; in other words, the four principal right ideals generated by them are isomorphic as right $R$-modules. This exercise was intended for prospective use toward the proofs of Theorem 60 and Theorem 61 on Baer *-rings in [Ka: pp.91-96]. For his Exercise 6 on p. 25, Kaplansky kindly offered his readers a "Hint". However, rendered with the author's trademark brevity, this "Hint" was itself no less than another substantial exercise. In all fairness, a full solution of Kaplansky's "Exercise 6" proved to be a considerable challenge by any yardstick.

That was the situation about fifty years ago, in 1967-68. Nowadays, with so much more known about idempotents and idempotent identities in rings, a rather natural new solution can be given for Kaplansky's "Exercise 6". More remarkably, the theme of this exercise can be further developed so as to give a full-fledged characterization theorem for the rings $R$ that appeared in the exercise. To explain this from a more general point of view, we'll use the notation $[x, y]$ for the commutator $x y-y x$, and the notation $\langle x, y\rangle$ for the anti-commutator $x y+y x$, for any two elements $x, y$ in a ring $R$. For the main purposes of this paper, it is convenient to introduce the following two ring-theoretic properties, where idem $(R)$ will henceforth denote the set of idempotents in the ring $R$, and $\mathrm{U}(R)$ will denote the group of units of $R$.

Property K : There exist $e, e^{\prime} \in \operatorname{idem}(R)$ such that $\left[e, e^{\prime}\right] \in \mathrm{U}(R)$.

Property $\overline{\mathbf{K}}$ : There exist $e, e^{\prime} \in \operatorname{idem}(R)$ such that $\left\langle e, e^{\prime}\right\rangle \in \mathrm{U}(R)$. 
The easiest class of rings to deal with in the investigation of these properties is the class of abelian rings; that is, rings in which all idempotents are central. It is easy to see that such a ring $R$ has Property $\mathrm{K}$ iff $R=0$, while it has Property $\overline{\mathrm{K}}$ iff $2 \in \mathrm{U}(R)$. (In fact, in view of the fact that $\langle 1,1\rangle=2$, any ring with $2 \in \mathrm{U}(R)$ has Property $\overline{\mathrm{K}}$.) Another easy observation we can make is that, if $R \rightarrow R^{\prime}$ is a (unital) ring homomorphism, then $R$ having one of the two properties above implies that $R^{\prime}$ has the same property, whereby any matrix ring $\mathbb{M}_{n}(R)$ will also have the same property.

The first two main results in this paper are Theorem A and Theorem B below, where the former (to be proved in $\S 2$ ) explains our choice of the notation $\overline{\mathrm{K}}$ since it has the obvious consequence that the class of rings with Property $\mathrm{K}$ is (properly) contained in the class of rings with Property $\overline{\mathrm{K}}$.

Theorem A. For any $e, e^{\prime} \in \operatorname{idem}(R),\left[e, e^{\prime}\right] \in \mathrm{U}(R)$ implies that $\left\langle e, e^{\prime}\right\rangle \in \mathrm{U}(R)$ (though not conversely). In particular, if $R$ has Property $\mathrm{K}$, then it has Property $\overline{\mathrm{K}}$.

Theorem B. A ring $R$ has Property $\mathrm{K}$ iff $R \cong \mathbb{M}_{2}(S)$ for a ring $S$ in which $1 \in$ $\mathrm{U}(S)+\mathrm{U}(S)$.

From our more general perspective, the solution of Kaplansky's Exercise 6 is just a part of the work needed for proving the "only if" part of Theorem B. In $\S 3$, this theorem is proved by using judiciously various idempotent identities of Nicholson [Ni], Kato [Kt], and Koliha-Rakočević $\left[\mathrm{KR}_{1}, \mathrm{KR}_{2}\right]$, which we first develop ad hoc in $\S 2$. The classical notion of Bott-Duffin invertibility (relative to an idempotent) introduced in [BD] turns out to play a significant role in proving Theorem B, so the basic ingredients of the Bott-Duffin theory are briefly recalled at the beginning of $\S 2$ as well.

With Theorem A and Theorem B at our disposal, a natural question to ask is when would a matrix ring $\mathbb{M}_{n}(T)$ (over a given type of base rings $T$ ) have Property $\mathrm{K}$ or Property $\overline{\mathrm{K}}$. For instance, in the case where $T$ is a division ring, this would amount to asking which simple artinian rings would have Property $\mathrm{K}$ or Property $\overline{\mathrm{K}}$. Indeed, taking $T$ to be a local ring or a nonzero commutative ring, we have the following result in $\S 3$ on matrix rings $\mathbb{M}_{n}(T)$ with Property $\mathrm{K}$.

Theorem C. Let $R=\mathbb{M}_{n}(T)$, where $T$ is a local ring or a nonzero commutative ring. Then $R$ has Property K iff either $n \in\{4,6,8, \ldots\}$, or $n=2$ and $1_{T} \in \mathrm{U}(T)+\mathrm{U}(T)$. (Note that in the case where $(T, \mathfrak{m})$ is local ring, the condition $1_{T} \in \mathrm{U}(T)+\mathrm{U}(T)$ would amount to the simpler statement that $|T / \mathfrak{m}|>2$.)

As for Property $\bar{K}$, the corresponding result (to be proved in $§ 4$ ) is the following.

Theorem D. Let $T$ be a local ring or a commutative ring. Then $R=\mathbb{M}_{n}(T)$ has Property $\overline{\mathrm{K}}$ iff we are in one of the following cases:

(1) $n \in\{4,6,8, \ldots\}$.

(2) $n=2$ and $1_{T} \in \mathrm{U}(T)+\mathrm{U}(T)$.

(3) $n$ is odd and $2 \in \mathrm{U}(T)$. 
As a spin-off of our investigations on invertible commutators of the form $\left[e, e^{\prime}\right]$ where $e, e^{\prime} \in \operatorname{idem}(R)$, we also consider the case where $e^{\prime}$ is allowed to be an arbitrary element of $R$, while $e$ is replaced by an element with some specific property "comparable" to idempotency. Working with these more general commutators (and anti-commutators) and building on the work of Fuchs-Maxson-Pilz in [FMP: (III.2)], we obtain in $\S 5$ a number of new criteria for $2 \times 2$ matrix rings, three of which are summarized as follows.

Theorem E. A ring $R$ is a $2 \times 2$ matrix ring over some other ring iff there is an invertible commutator $[e, *]$ where $e^{2}=e$, iff there is an invertible commutator $[p, *]$ where $p^{2}=0$, iff there is an invertible commutator $[u, *]$ where $u^{2}=1$.

The terminology and notations introduced so far in this Introduction will be used freely throughout the paper. For any $\operatorname{ring} R, \operatorname{rad}(R)$ denotes the Jacobson radical of $R$, and the words "exchange ring" will be used in the sense of Warfield [Wa] and Nicholson [Ni]. By saying that a matrix $M \in \mathbb{M}_{n}(T)$ is diagonalizable, we'll mean that $M$ is similar to a diagonal matrix in $\mathbb{M}_{n}(T)$. Other standard terminology and conventions in ring theory follow mainly those in $[\mathrm{Go}],\left[\mathrm{La}_{1}\right]$, and $\left[\mathrm{La}_{3}\right]$.

\section{§2. Idempotent Identities of Kato and Koliha-Rakočević}

One key ingredient used in this beginning section is the basic notion of Bott-Duffin invertibility introduced in the early paper [BD]. In order to give a relatively self-contained exposition of our results, we'll start by recalling some definitions and facts from [BD]. For any idempotent $e$ in a ring $R$, an element $a \in R$ is said to be Bott-Duffin invertible relative to $e$ if $e a e \in \mathrm{U}(e R e)$. In this case, the inverse of eae in the corner ring $e R e$ is said to be the Bott-Duffin inverse of $a$ relative to $e$. For the sake of completeness, we state and prove the following classical characterization result for Bott-Duffin invertibility.

Theorem 2.1. For $a \in R$ and $e \in \operatorname{idem}(R)$, the following are equivalent:

(1) a is Bott-Duffin invertible relative to $e$.

(2) $1-e+a e \in \mathrm{U}(R) . \quad(3) 1-e+e a \in \mathrm{U}(R)$.

(4) $1-e+e a e \in \mathrm{U}(R)$.

Proof. For any ring $R$, Jacobson's Lemma (see, e.g. [La, : Exercise 1.6]) states that, for any $x, y \in R, 1-x y \in \mathrm{U}(R)$ iff $1-y x \in \mathrm{U}(R)$. Since $1-e+a e=1-(1-a e) e$, this Lemma gives $(2) \Leftrightarrow(4)$. By left-right symmetry, we have also $(3) \Leftrightarrow(4)$. To see that $(4) \Leftrightarrow(1)$, let $f=1-e$. With respect to the idempotent $e$, the element $f+e a e$ has a diagonal Peirce decomposition matrix $\left(\begin{array}{cc}e a e & 0 \\ 0 & f\end{array}\right)$. Such a matrix is invertible iff $e a e \in \mathrm{U}(e R e)$, which is the defining condition for (1). This shows that $(4) \Leftrightarrow(1)$.

Next, we state the following key result from Kato's book [Kt: (I.4.34), (I.4.44)].

Proposition 2.2. (Kato's Identities) For any $e, e^{\prime} \in \operatorname{idem}(R)$, we have

$$
\left(e-e^{\prime}\right)^{2}+\left(1-e-e^{\prime}\right)^{2}=1,
$$

which amounts to

$$
\left(e-e^{\prime}\right)^{2}=\left(e+e^{\prime}\right)\left(2-e-e^{\prime}\right)
$$


where the two factors on the RHS commute. Also, writing $r=1-e+e^{\prime} e$ and $s=$ $1-e^{\prime}+e e^{\prime}$, we have

$$
r s=s r=\left(1+e-e^{\prime}\right)\left(1-e+e^{\prime}\right)=\left(1-e-e^{\prime}\right)^{2} .
$$

Proof. The identity (2.3) is easily verified by a direct expansion of the LHS. By transposing the term $\left(1-e-e^{\prime}\right)^{2}$ to the RHS and using the factorization $1-x^{2}=(1-x)(1+x)$, we get the identity (2.4). Similarly, by transposing the term $\left(e-e^{\prime}\right)^{2}$ in (2.3) to the RHS and using the factorization $1-y^{2}=(1+y)(1-y)$, we get the last equality in (2.5). The first two equalities in (2.5) are easily verified by direct expansions.

Corollary 2.6. Two idempotents $e, e^{\prime} \in R$ are Bott-Duffin invertible relative to each other iff $1-e-e^{\prime} \in \mathrm{U}(R)$.

Proof. This follows from (2.5), applied in conjunction with Theorem 2.1.

Remark 2.7. In general, $e^{\prime}$ being Bott-Duffin invertible relative to $e$ alone does not imply that $e$ is Bott-Duffin invertible relative to $e^{\prime}$. For instance, if $e^{\prime}=1 \neq e$, then $e^{\prime}$ is Bott-Duffin invertible relative to $e$, but $e$ is not Bott-Duffin invertible relative to $e^{\prime}$. In this example, we have $1-e-e^{\prime}=-e \notin \mathrm{U}(R)$.

In $\left[\mathrm{KR}_{1}\right.$ : Theorem 3.5], it was proved that, for any idempotents $e, e^{\prime}$ in any ring, $e-e^{\prime} \in \mathrm{U}(R)$ iff $e+e^{\prime} \in \mathrm{U}(R)$ and $1-e e^{\prime} \in \mathrm{U}(R)$. (The hypothesis $2 \in \mathrm{U}(R)$ was included in the statement of this theorem, but the result can be seen to be true without such a hypothesis.) Using here the crucial identity (2.4), we'll prove the following closely related result.

Theorem 2.8. For any $e, e^{\prime} \in \operatorname{idem}(R)$, we have $e-e^{\prime} \in \mathrm{U}(R)$ iff $e+e^{\prime} \in \mathrm{U}(R)$ and $f+f^{\prime} \in \mathrm{U}(R)$, where $f=1-e$ and $f^{\prime}=1-e^{\prime}$. In this case, $e$ is similar to $1-e^{\prime}$.

Proof. The "iff" statement follows from the identity (2.4) (since $2-e-e^{\prime}=f+f^{\prime}$ ). The last statement is a special case of a result of Nicholson [Ni: Proposition 1.8] on the clean representations of elements in a ring. To make our exposition self-contained, we recall Nicholson's proof (in our special case). If $u:=e-e^{\prime} \in \mathrm{U}(R)$, then

$$
\left(1-e^{\prime}\right) u=\left(1-e^{\prime}\right)\left(e-e^{\prime}\right)=\left(1-e^{\prime}\right) e=\left(e-e^{\prime}\right) e=u e .
$$

Thus, $e=u^{-1}\left(1-e^{\prime}\right) u$ is similar to $1-e^{\prime}$.

Remark 2.9. In general, $e+e^{\prime} \in \mathrm{U}(R)$ alone need not imply that $e-e^{\prime} \in \mathrm{U}(R)$. For instance, in any nonzero ring $R$ in which $2 \in \mathrm{U}(R)$, the idempotents $e=e^{\prime}=1$ have the property that $e+e^{\prime}=2 \in \mathrm{U}(R)$, but $e-e^{\prime}=0 \notin \mathrm{U}(R)$.

Next we give a systematic derivation for two useful identities on the commutator $\left[e, e^{\prime}\right]=e e^{\prime}-e^{\prime} e$ and the anti-commutator $\left\langle e, e^{\prime}\right\rangle=e e^{\prime}+e^{\prime} e$ from $\left[\mathrm{KR}_{2}:\right.$ p. 103] and [KR ${ }_{1}$ : p. 289]. 
Proposition 2.10. (Koliha-Rakočević Identities) For any $e, e^{\prime} \in \operatorname{idem}(R)$ and $f=1-e$, we have

$$
\begin{aligned}
& {\left[e, e^{\prime}\right]=\left(e-e^{\prime}\right)\left(e^{\prime}-f\right)=\left(f-e^{\prime}\right)\left(e-e^{\prime}\right) ;} \\
& \left\langle e, e^{\prime}\right\rangle=\left(e+e^{\prime}\right)\left(e^{\prime}-f\right)=\left(e^{\prime}-f\right)\left(e+e^{\prime}\right) .
\end{aligned}
$$

Proof. From $\left(e+e^{\prime}\right)^{2}=e+e^{\prime}+\left\langle e, e^{\prime}\right\rangle$, transposition of the term $e+e^{\prime}$ gives the two equalities in (2.12). Similarly, from $\left(e-e^{\prime}\right)\left(e+e^{\prime}\right)=e-e^{\prime}+\left[e, e^{\prime}\right]$, transposition of the term $e-e^{\prime}$ gives the first equality in (2.11), and the second equality follows by working instead with $\left(e+e^{\prime}\right)\left(e-e^{\prime}\right)=e-e^{\prime}-\left[e, e^{\prime}\right]$.

Kaplansky asserted in [Ka: p. 25, Exercise 6] that, under the assumption $\left[e, e^{\prime}\right] \in$ $\mathrm{U}(R)$, the idempotents $e, e^{\prime} \in R$ are Bott-Duffin invertible relative to each other. With the help of Proposition 2.10, one can prove the more precise result in (1) below, and by the same token, prove also its complete analogue in (2) for anti-commutators.

Theorem 2.13. For any $e, e^{\prime} \in \operatorname{idem}(R)$ and $f=1-e$, the following hold.

(1) $\left[e, e^{\prime}\right] \in \mathrm{U}(R)$ iff $e-e^{\prime} \in \mathrm{U}(R)$ and $f-e^{\prime} \in \mathrm{U}(R)$, iff $e-e^{\prime} \in \mathrm{U}(R)$ and $e, e^{\prime}$ are Bott-Duffin invertible relative to each other.

(2) $\left\langle e, e^{\prime}\right\rangle \in \mathrm{U}(R)$ iff $e+e^{\prime} \in \mathrm{U}(R)$ and $f-e^{\prime} \in \mathrm{U}(R)$, iff $e+e^{\prime} \in \mathrm{U}(R)$ and $e, e^{\prime}$ are Bott-Duffin invertible relative to each other.

(3) $\left[e, e^{\prime}\right] \in \mathrm{U}(R)$ implies that $\left\langle e, e^{\prime}\right\rangle \in \mathrm{U}(R)$.

Proof. For (1), the first "iff" statement follows from (2.11); see [ $\left.\mathrm{KR}_{2}:(3.6)\right]$. The second "iff" statement then follows from Corollary 2.6. (2) is proved similarly by using (2.11) and Corollary 2.6 (although the first "iff" statement in $(2)$ was stated in $\left[\mathrm{KR}_{1}\right.$ : Theorem 3.6] with an extra assumption that $2 \in \mathrm{U}(R)$ ). Finally, (3) follows from (1) and (2), since $e-e^{\prime} \in \mathrm{U}(R) \Rightarrow e+e^{\prime} \in \mathrm{U}(R)$ according to Theorem 2.8.

Recalling from $\S 1$ that "Property K" (respectively, "Property $\overline{\mathrm{K}}$ ") on a ring $R$ means the existence of $e, e^{\prime} \in \operatorname{idem}(R)$ such that $\left[e, e^{\prime}\right] \in \mathrm{U}(R)$ (respectively, $\left\langle e, e^{\prime}\right\rangle \in \mathrm{U}(R)$ ), we can draw the following conclusions from Theorem 2.13.

Corollary 2.14. If a ring $R$ has Property $\mathrm{K}$, then it has Property $\overline{\mathrm{K}}$. However, the converse of this statement fails in general.

Proof. The first statement here follows from part (3) of Theorem 2.13. The second statement follows from the observation (made in $\S 1$ ) that any ring $R$ with $2 \in \mathrm{U}(R)$ has Property $\overline{\mathrm{K}}$, while an abelian ring $R \neq 0$ cannot have Property $\mathrm{K}$.

For future reference, we record in the following a useful result on the Jacobson radical and an immediate consequence thereof.

Proposition 2.15. Let $J \subseteq \operatorname{rad}(R)$ be an ideal of $R$ such that idempotents lift modulo $J$. Then $R$ has Property $\mathrm{K}$ iff $R / J$ does. The same statement holds for Property $\overline{\mathrm{K}}$. 
Proof. As we have observed in $\S 1$, the "only if" part holds without any assumptions on the ideal $J$. In the case where idempotents lift modulo $J \subseteq \operatorname{rad}(R)$, the "if" part follows from the observation that an element $s \in R$ is a unit iff $\bar{s}$ is a unit in $\bar{R}=R / J$.

Corollary 2.16. Let $J$ be an ideal of $R$ such that one of the following holds:

(1) $J$ is a nil ideal.

(2) $J \subseteq \operatorname{rad}(R)$ and $R$ is an exchange ring.

Then the conclusions of Proposition 2.15 hold for the two rings $R$ and $R / J$.

Proof. (1) If $J$ is a nil ideal, we have $J \subseteq \operatorname{rad}(R)$ by [La $:$ (4.11)]. On the other hand, by [La $:$ (21.28)], idempotents lift modulo $J$. Thus, Proposition 2.15 applies.

(2) If $R$ is an exchange ring, idempotents can be lifted modulo any ideal in $R$ (according to $[\mathrm{Ni}]$ ), so again Proposition 2.15 applies.

We shall conclude the discussions in this section by using Corollary 2.16 to prove the following two easy results on upper triangular rings.

Proposition 2.17. Let $S, T$ be two rings, and let $M$ be any $(S, T)$-bimodule. Then the formal triangular ring $R=\left(\begin{array}{cc}S & M \\ 0 & T\end{array}\right)$ has Property $\mathrm{K}$ (respectively, Property $\overline{\mathrm{K}}$ ) iff $S$ and $T$ both do.

Proof. The "only if" part is clear, since $S$ and $T$ can both be thought of as factor rings of $R$. Conversely, assume that $S$ and $T$ have Property K. Then $S \times T$ also does. As $J:=\left(\begin{array}{cc}0 & M \\ 0 & 0\end{array}\right)$ is a square-zero ideal in $R$, and $R / J \cong S \times T$, it follows from Case (1) of Corollary 2.16 that $R$ also has Property K. The case where $S$ and $T$ both have Property $\overline{\mathrm{K}}$ is similar.

In the case of $n \times n$ upper triangular matrices over a given ring $S$, the ideal $J$ of strictly upper triangular matrices has the property that $J^{n}=0$. Thus, the same method of proof (using Case (1) of Corollary 2.16) yields the following similar result.

Proposition 2.18. Let $R=\mathbb{T}_{n}(S)$ be the ring of $n \times n$ upper triangular matrices over a ring $S$. Then $R$ has Property K (respectively, Property $\overline{\mathrm{K}}$ ) iff $S$ does.

\section{§3. Rings with Property K}

To study rings with the Property K, we begin by giving a streamlined proof for the rest of Kaplansky's "Exercise 6" from [Ka: p. 25]; see also Theorem 60 in [Ka: p. 96]. The proof to be given below is in some sense much easier to grasp than that given by Kaplansky since it makes full use of the efficient tools developed in $\S 2$.

Theorem 3.1. If $e, e^{\prime} \in \operatorname{idem}(R)$ are such that $\left[e, e^{\prime}\right] \in \mathrm{U}(R)$, then the following hold.

(1) The four idempotents $e, e^{\prime}, 1-e, 1-e^{\prime}$ are pairwise similar.

(2) $R=e R \oplus e^{\prime} R=R e \oplus R e^{\prime}$. 
Proof. (1) Let $f=1-e$. By Theorem 2.13(1), we have $e-e^{\prime} \in \mathrm{U}(R)$ and $f-e^{\prime} \in \mathrm{U}(R)$. Therefore, by the last part of Theorem 2.8, $e^{\prime}$ is similar to $f$ as well as to $e$. By symmetry, it follows that $e, e^{\prime}, 1-e, 1-e^{\prime}$ are pairwise similar.

(2) By symmetry, it suffices to prove that $R=e R \oplus e^{\prime} R$. Since $e-e^{\prime} \in \mathrm{U}(R)$, clearly $e R+e^{\prime} R=R$. Also, for any $x \in e R \cap e^{\prime} R$, we have $x=e x=e^{\prime} x$. Thus, $\left(e-e^{\prime}\right) x=0$, and hence $x=0$. This proves that $R=e R \oplus e^{\prime} R$. (In conclusion, we note that (2) can also be deduced as a consequence of $\left[\mathrm{KR}_{2}\right.$ : Theorem 3.2].)

Remark 3.2. In general, if two idempotents $e, e^{\prime}$ in a ring $R$ are such that $e, e^{\prime}$, $1-e, 1-e^{\prime}$ are pairwise similar, the commutator $\left[e, e^{\prime}\right]$ need not be a unit in $R$, and $e^{\prime}$ need not be Bott-Duffin invertible relative to $e$. For instance, if $R=\mathbb{M}_{2}(S)$ over a nonzero ring $S$ and $E_{i j}$ are the matrix units, then $e=E_{11}$ and $e^{\prime}=E_{22}$ certainly have the similarity properties mentioned above, but $\left[e, e^{\prime}\right]=0$, and $e e^{\prime} e=0$ (so $e^{\prime}$ is not Bott-Duffin invertible relative to $e$ ). On the other hand, if we have only $[e, r] \in \mathrm{U}(R)$ where $r \in R$ (or even $r \in \mathrm{U}(R)$ ), it also does not follow that $r$ is Bott-Duffin invertible relative to $e$, as is shown by the example $e=E_{11}$ and $r=E_{12}+E_{21}$, for which $[e, r]=E_{12}-E_{21} \in \mathrm{U}(R)$, but again ere $=0$.

Now we are in a position to prove Theorem B stated in the Introduction. The remarkable thing about this theorem is that a single commutator property on a ring turns out to be enough to determine the structure of the whole ring. Just for the record, we note that it was Kaplansky's "Exercise 6" in [Ka: p. 25] which had given us the impetus to prove the "only if" part of this theorem.

Theorem 3.3. A ring $R$ has Property $\mathrm{K}$ iff $R \cong \mathbb{M}_{2}(S)$ for a ring $S$ in which $1 \in \mathrm{U}(S)+\mathrm{U}(S)$.

Proof. First assume that $1=a+b \in S$ where $a, b \in \mathrm{U}(S)$. In the matrix ring $\mathbb{M}_{2}(S)$, we check easily that $e=\left(\begin{array}{ll}a & b \\ a & b\end{array}\right)$ is an idempotent, and that the commutator $\left[e, E_{11}\right]=\left(\begin{array}{cc}0 & -b \\ a & 0\end{array}\right)$ is a unit. Thus, $\mathbb{M}_{2}(S)$ has Property K. (In testimony to the implication statement in Theorem 2.13(3), the anti-commutator $\left\langle e, E_{11}\right\rangle=\left(\begin{array}{cc}2 a & b \\ a & 0\end{array}\right)$ is indeed a unit too, so the ring $\mathbb{M}_{2}(S)$ also has Property $\overline{\mathrm{K}}$ as expected.)

Conversely, if a ring $R$ has Property $\mathrm{K}$, fix two idempotents $e, e^{\prime} \in R$ such that $\left[e, e^{\prime}\right] \in \mathrm{U}(R)$. By Theorem 3.1, $f:=1-e$ is similar to $e$. This implies that $f R \cong e R$, and so $R_{R}=e R \oplus f R \cong e R \oplus e R$. Taking endomorphism rings, we have

$$
R \cong \operatorname{End}_{R}(e R \oplus e R) \cong \mathbb{M}_{2}(S)
$$

where $S:=\operatorname{End}_{R}(e R)$ can be identified with the corner ring $e R e$. By Theorem 2.13(1), $e e^{\prime} e \in \mathrm{U}(S)$. Similarly, for $f^{\prime}:=1-e^{\prime}$, the fact that $\left[e, f^{\prime}\right]=-\left[e, e^{\prime}\right] \in \mathrm{U}(R)$ implies that $e f^{\prime} e \in \mathrm{U}(S)$ too. Thus, $1_{S}=e=e e^{\prime} e+e f^{\prime} e \in \mathrm{U}(S)+\mathrm{U}(S)$, as desired.

In connection with the condition $1 \in \mathrm{U}(S)+\mathrm{U}(S)$ appearing in Theorem 3.3, we should point out that it is in fact equivalent to the following ostensibly stronger condition: 
for any integer $n \geq 1$, any unit in $S$ is a sum of $n$ units in $S$. In the case where $S$ is a unit-regular ring, the condition $1_{S} \in \mathrm{U}(S)+\mathrm{U}(S)$ can also be self-strengthened into $S=\mathrm{U}(S)+\mathrm{U}(S)$, according to [GW: Theorem 3.8]. In the following, we will state two interesting consequences of Theorem 3.3.

Corollary 3.4. A ring $R$ has Property $\mathrm{K}$ with $2 \in \mathrm{U}(R)$ iff $R \cong \mathbb{M}_{2}(S)$ for some ring $S$ with $2 \in \mathrm{U}(S)$.

Proof. The "if" part follows from Theorem 3.3 since $2 \in \mathrm{U}(S)$ implies that $1=$ $2^{-1}+2^{-1} \in \mathrm{U}(S)+\mathrm{U}(S)$, as well as $2 \in \mathrm{U}\left(\mathbb{M}_{2}(S)\right)$. Conversely, if a ring $R$ has Property $\mathrm{K}$ along with $2 \in \mathrm{U}(R)$, Theorem 3.3 implies that $R \cong \mathbb{M}_{2}(S)$ for some ring $S$. Clearly, the fact that $2 \in \mathrm{U}(R)$ implies that $2 \in \mathrm{U}(S)$.

A second application of the "if" part of Theorem 3.3 is that it gives a good supply of examples of matrix rings having the Property K, as follows.

Corollary 3.5. If $R=\mathbb{M}_{2 m}(T)$ (for any ring $T$ ) where $m \geq 2$, or $R=\operatorname{End}_{D}(V)$ for some infinite-dimensional right vector space $V$ over a division ring $D$, then $R$ has Property K.

Proof. First assume that $R=\mathbb{M}_{2 m}(T)$ where $m \geq 2$. By a result of Henriksen $[\mathrm{He}]$, the identity matrix $I_{m}$ is a sum of two units in $\mathbb{M}_{m}(T)$. Thus, by Theorem 3.3, $R \cong \mathbb{M}_{2}\left(\mathbb{M}_{m}(T)\right)$ has Property K. Finally, assume that $R=$ End $\left(V_{D}\right)$ as in the statement of the Corollary. By [La, Example 1.4], we have $R \cong \mathbb{M}_{n}(R)$ for every $n \geq 1$. Applying this for $n=4$ (for instance), we are back to the case treated above.

Example 3.6. In general, if $T$ is a ring such that $R:=\mathbb{M}_{2}(T)$ has Property $\mathrm{K}$, Theorem 3.3 only says that $R \cong \mathbb{M}_{2}(S)$ for some ring $S$ in which 1 is a sum of two units. Since $T$ may not be isomorphic to $S$, this does not imply that $1_{T} \in \mathrm{U}(T)+\mathrm{U}(T)$. An explicit example to illustrate the possible failure of $1_{T} \in \mathrm{U}(T)+\mathrm{U}(T)$ is as follows. For any field $k$, we construct after Leavitt [Le] and Cohn $\left[\mathrm{Co}_{1}\right]$ a $k$-algebra $T$ with a generic invertible $3 \times 2$ matrix. This matrix defines a right $T$-module isomorphism $T^{2} \cong T^{3}$, which induces another isomorphism $T^{2} \cong T^{4}$. Taking $T$-endomorphism rings gives a ring isomorphism $R:=\mathbb{M}_{2}(T) \cong \mathbb{M}_{4}(T)$. Thus, it follows from Corollary 3.5 that $R$ has Property K. By a recent result of $\mathrm{G}$. Bergman $\left[\mathrm{Be}_{2}\right]$ based on his earlier work $\left[\mathrm{Be}_{1}\right], \mathrm{U}(T)=\mathrm{U}(k)$. In particular, if we take $k$ to be the field of two elements, we'll have $\mathrm{U}(T)=\{1\}$, in which case clearly $1_{T} \notin \mathrm{U}(T)+\mathrm{U}(T)$.

Example 3.6 leads naturally to the following

Question 3.7. If $T$ is a ring such that $\mathbb{M}_{2}(T)$ has Property $\mathrm{K}$, under what additional assumptions on $T$ can we conclude that $1_{T} \in \mathrm{U}(T)+\mathrm{U}(T)$ ?

The difficulty in dealing with this question stems mainly from the fact that $1_{T} \in$ $\mathrm{U}(T)+\mathrm{U}(T)$ is not a Morita invariant property of rings. Nevertheless, there are many classes of rings $T$ for which we can answer Question 3.7 in a satisfactory way. First, recall from $\left[\mathrm{La}_{3}: \S 17 \mathrm{C}\right]$ that a ring $T$ is said to be $\mathbb{M}_{\boldsymbol{n}}$-unique if, for any ring $S$, 
$\mathbb{M}_{n}(T) \cong \mathbb{M}_{n}(S)$ implies that $T \cong S$. Taking stock in this definition, we do have the following partial positive answer to Question 3.7.

Theorem 3.8. Let $R=\mathbb{M}_{2}(T)$ where $T$ satisfies one of the following conditions:

(1) $T$ is $\mathbb{M}_{2}$-unique.

(2) $T$ is an abelian ring and every idempotent in $R$ is diagonalizable.

Then $R$ has Property $\mathrm{K}$ iff $1_{T} \in \mathrm{U}(T)+\mathrm{U}(T)$.

Proof. The "if" part is true without any assumptions on $T$ by Theorem 3.3. The "only if" part in the two cases (1) and (2) will be handled separately, as follows.

Case (1). If $R$ has Property K, Theorem 3.3 implies that $R \cong \mathbb{M}_{2}(S)$ for some ring $S$ with $1_{S} \in \mathrm{U}(S)+\mathrm{U}(S)$. Given the $\mathbb{M}_{2}$-unique assumption in Case (1), we have $T \cong S$, and so $1_{T} \in \mathrm{U}(T)+\mathrm{U}(T)$.

Case (2). If $R$ has Property $\mathrm{K}$, fix a commutator $\left[e, e^{\prime}\right] \in \mathrm{U}(R)$ with $e, e^{\prime} \in \operatorname{idem}(R)$. Given the hypothesis in this case, we may assume (after a conjugation) that $e^{\prime}=$ $\operatorname{diag}(s, t)$, where $s, t \in T$ are necessarily central idempotents. Writing $e=\left(\begin{array}{ll}a & b \\ c & d\end{array}\right)$, we have $\left[e, e^{\prime}\right]=\left(\begin{array}{cc}0 & b(t-s) \\ c(s-t) & 0\end{array}\right) \in \mathrm{U}(R)$, which implies that $b, c \in \mathrm{U}(T)$. From the equation $e^{2}=e$, we get $a^{2}+b c=a$, so $a, 1-a$ are units in $T$, with sum 1 .

Having proved Theorem 3.8, we will mention in (3.9) - (3.13) below some of the more important classes of base rings $T$ to which the theorem can be applied.

(3.9) The most obvious class of examples of a $\operatorname{ring} T$ satisfying the hypothesis (2) of Theorem 3.8 is given by the projective-free rings defined by P. M. Cohn in $\left[\mathrm{Co}_{2}, \mathrm{Co}_{3}\right]$ : a ring $T$ is projective-free if every finitely generated projective right module over $T$ is free of a unique rank. (This notion is known to be left-right symmetric; see $\left[\mathrm{Co}_{2}\right]$.) Clearly, such a ring $T$ has only trivial idempotents (so it is abelian), and any idempotent in $\mathbb{M}_{n}(T)$ is similar to a diagonal matrix with 0's and 1's on the diagonal.

(3.10) Another example of a ring satisfying the hypothesis (2) in Theorem 3.8 is an abelian (von Neumann) regular ring $T$. The fact that any idempotent matrix over a regular ring is diagonalizable can be shown in at least two ways: first by using the refinement theorem (for finitely generated projective modules) of Goodearl and Handelman in [GH: (3.8)], and second, by using [Go: (2.6)] in conjunction with [SG: Theorem 9].

(3.11) In the formulation of "Open Problem (47)" in Goodearl's book [Go: p. 349], there is an extensive list of different classes of regular rings $T$ that are classically known to be $\mathbb{M}_{n}$-unique for all $n$. Thus, Theorem 3.8 is applicable to all such regular rings, under the hypothesis (1). The two best known classes among those mentioned in [Go: p. 349] are right self-injective regular rings [Go: (10.35)], and all regular rings whose primitive factors are artinian [Go: (6.12)]. (The latter class includes all abelian regular rings, which we have already mentioned in (3.10).) But unfortunately, unit-regular rings are not known to be $\mathbb{M}_{n}$-unique (even for $n=2$ ), so the answer to Question 3.7 has so far remained unknown in the case where the base $\operatorname{ring} T$ is unit-regular. 
(3.12) According to $\left[\mathrm{La}_{3}:(17.26)\right]$, any semilocal ring $T$ is $\mathbb{M}_{n}$-unique for all $n$. Thus, Theorem 3.8 is applicable to $T$ again under the hypothesis (1). In the special case where $(T, \mathfrak{m})$ is a local ring, it would be projective-free as well. In this case, as we have noted in the statement of Theorem B in $\S 1$, the conclusion $1_{T} \in \mathrm{U}(T)+\mathrm{U}(T)$ in Theorem 3.8 would amount to the simpler statement that $|T / \mathfrak{m}|>2$.

(3.13) Theorem 3.8 is applicable to any commutative ring $T$ (under hypothesis (1)) since $T$ is also known to be $\mathbb{M}_{n}$-unique (for all $n$ ) by $\left[\mathrm{La}_{3}:(17.31)\right]$. In this case, the conclusion of Theorem 3.8 is capable of another more concrete derivation, as follows. If $e, e^{\prime}$ are idempotents in $R=\mathbb{M}_{2}(T)$ with $\left[e, e^{\prime}\right] \in \mathrm{U}(R)$, it is easy to show using [KLS: Formula (1.1)] that $\operatorname{tr}\left(e e^{\prime}\right)$ and $1-\operatorname{tr}\left(e e^{\prime}\right)$ are both units in $T$, with sum 1.

Using Theorem 3.3 and Corollary 3.5, it is now easy to determine all matrix rings over local rings and nonzero commutative rings that have Property $\mathrm{K}$.

Theorem 3.14. Let $T$ be a local ring or a nonzero commutative ring. Then $R:=$ $\mathbb{M}_{n}(T)$ has Property $\mathrm{K}$ iff either $n \in\{4,6,8, \ldots\}$, or $n=2$ and $1_{T} \in \mathrm{U}(T)+\mathrm{U}(T)$.

Proof. The "if" part follows from Theorem 3.3 and Corollary 3.5. For the "only if" part, assume that $R=\mathbb{M}_{n}(T)$ has Property K. Since $T$ is a local ring or a nonzero commutative ring, there exists an ideal $\mathfrak{m} \subseteq T$ such that $T / \mathfrak{m}$ is a division ring. Then $\mathbb{M}_{n}(T / \mathfrak{m}) \cong \mathbb{M}_{n}(T) / \mathbb{M}_{n}(\mathfrak{m})$ also has Property K. Applying Theorem 3.3, we have $\mathbb{M}_{n}(T / \mathfrak{m}) \cong \mathbb{M}_{2}(S)$ for some ring $S$ with $1_{S} \in \mathrm{U}(S)+\mathrm{U}(S)$. By the uniqueness part of Wedderburn's theorem, we see that $n$ must be even. Finally, in the special case $n=2, \quad R=\mathbb{M}_{2}(T)$ having Property $\mathrm{K}$ would imply that $1_{T} \in \mathrm{U}(T)+\mathrm{U}(T)$ by the remarks in (3.12) and (3.13).

Example 3.15. Let $R=\mathbb{M}_{n}(D)$ be a typical simple artinian ring, where $D$ is a division ring. According to Theorem 3.14, $R$ has Property $\mathrm{K}$ except precisely when $n$ is odd or when $n=2$ and $|D|=2$.

Example 3.16. Let $\mathbb{Z}_{(p)}$ denote the localization of $\mathbb{Z}$ at the prime ideal $(p)$. If $T=\mathbb{Z}$ or $\mathbb{Z}_{(2)}, \mathbb{M}_{n}(T)$ has Property $\mathrm{K}$ iff $n \in\{4,6,8, \ldots\}$. On the other hand, if $T=\mathbb{Z}_{(p)}$ where $p$ is an odd prime, then $\mathbb{M}_{n}(T)$ has Property $\mathrm{K}$ iff $n$ is even.

Example 3.17. If the ring $T$ in Theorem 3.14 is allowed to be noncommutative and not a local ring, the "only if" part in the theorem may no longer hold. For instance, we may take $T$ to be any nonzero ring with Property $\mathrm{K}$ (so $T$ is necessarily noncommutative). Then for any $n \geq 1$ (odd or even), $R=\mathbb{M}_{n}(T)$ also has Property $\mathrm{K}$ since $T$ does.

\section{§4. Interplay Between Property $K$ and Property $\bar{K}$}

In this section, we turn our attention to rings with Property $\overline{\mathrm{K}}$. Our goal is to understand more precisely the relationship and interaction between Property $\mathrm{K}$ and Property $\overline{\mathrm{K}}$. We begin by recalling that any ring $R$ with $2 \in \mathrm{U}(R)$ has Property $\overline{\mathrm{K}}$, but not necessarily Property $\mathrm{K}$. On the other hand, a $2 \times 2$ matrix ring such as $R=\mathbb{M}_{2}\left(\mathbb{F}_{2}\right)$ does not have Property $\mathrm{K}$ (e.g. by Theorem 3.3), and hence also does not have Property $\overline{\mathrm{K}}$ since $\operatorname{char}(R)=2$. From this example, it follows for instance that if 
$T$ is any ring with an ideal of index 2 , then $\mathbb{M}_{2}(T)$ (with factor ring $R$ ) does not have Property $\overline{\mathrm{K}}$, and hence also not Property $\mathrm{K}$.

Our first main result in this section is Theorem 4.1 below, where part (1) gives some necessary conditions on the invertibility of an anti-commutator $\left\langle e, e^{\prime}\right\rangle \in R$, while part (2) offers a somewhat unexpected characterization for the rings with Property $\overline{\mathrm{K}}$ without the use of products of idempotents.

Theorem 4.1. (1) If $e, e^{\prime} \in \mathrm{idem}(R)$ are such that $\left\langle e, e^{\prime}\right\rangle \in \mathrm{U}(R)$, then $e^{\prime}$ is similar to $e$, and we have $\langle e, w\rangle \in \mathrm{U}(R)$ for some $w \in \mathrm{U}(R)$.

(2) A ring $R$ has Property $\overline{\mathrm{K}}$ iff there exists a unit $u \in \operatorname{idem}(R)+\operatorname{idem}(R)$ such that $1-u \in \mathrm{U}(R)$.

Proof. (1) According to Theorem 2.13(2), $\left\langle e, e^{\prime}\right\rangle \in \mathrm{U}(R)$ amounts to two conditions:

$$
e+e^{\prime} \in \mathrm{U}(R) \text {, and } 1-e-e^{\prime} \in \mathrm{U}(R) .
$$

The latter implies (by Theorem 2.8) that $e^{\prime}=w e w^{-1}$ for some $w \in \mathrm{U}(R)$. Then $e+w e w^{-1}=e+e^{\prime} \in \mathrm{U}(R)$ implies that $\langle e, w\rangle=e w+w e=u w \in \mathrm{U}(R)$.

(2) If $R$ has Property $\overline{\mathrm{K}}$, there exist $e, e^{\prime} \in \operatorname{idem}(R)$ satisfying (4.2). Adding the two elements in (4.2) shows that 1 is a sum of two units, the first one of which is a sum of two idempotents in $R$. Conversely, if $1=u+u^{\prime}$ where $u, u^{\prime} \in \mathrm{U}(R)$ and $u=e+e^{\prime}$ for some $e, e^{\prime} \in \operatorname{idem}(R)$, then $1-e-e^{\prime}=1-u=u^{\prime} \in \mathrm{U}(R)$. Thus, (4.2) is satisfied, so $R$ has Property $\overline{\mathrm{K}}$.

Theorem 4.1 for rings with Property $\overline{\mathrm{K}}$ has a complete analogue for rings with Property $\mathrm{K}$ too, which we shall present below. One good reason we have chosen to prove Theorem 4.1 for Property $\overline{\mathrm{K}}$ first is that the proof of its part (2) is easier and more intuitive, while it gives an impetus toward finding an analogous (but somewhat harder) characterization result in the case of Property K.

Theorem 4.3. (1) If $e, e^{\prime} \in \operatorname{idem}(R)$ are such that $\left[e, e^{\prime}\right] \in \mathrm{U}(R)$, then we have $[e, v] \in \mathrm{U}(R)$ for some $v \in \mathrm{U}(R)$.

(2) A ring $R$ has Property $\mathrm{K}$ iff there exists a unit $v \in \operatorname{idem}(R)$ - idem $(R)$ such that $1 \pm v \in \mathrm{U}(R)$ for both signs.

Proof. (1) By Theorem 2.13(1), $\left[e, e^{\prime}\right] \in \mathrm{U}(R) \Rightarrow v:=e-e^{\prime} \in \mathrm{U}(R)$, and so

$$
[e, v]=\left[e, e-e^{\prime}\right]=-\left[e, e^{\prime}\right] \in \mathrm{U}(R) .
$$

The fact that one can "replace" the idempotent $e^{\prime}$ by a unit $v$ (while still achieving the property $[e, v] \in \mathrm{U}(R)$ ) reminds us somewhat of the theorem of de Sá as discussed in [Sa] and [KL], although our result here applies quite generally to any ring $R$, as long as $e, e^{\prime} \in \operatorname{idem}(R)$. (Needless to say, the converse of (1) fails in general. For instance, taking $R=\mathbb{M}_{2}(T)$ for any ring $T,[e, v]$ is an invertible commutator for $e=E_{11} \in \operatorname{idem}(R)$ and $v \in \mathrm{U}(R)$. But for $S=\mathbb{Z}$ or $\mathbb{F}_{2}$ for instance, we cannot find any invertible $\left[e_{1}, e_{2}\right]$ with $e_{1}, e_{2} \in \operatorname{idem}(R)$.) 
(2) First assume $R$ has Property $\mathrm{K}$ and fix an invertible commutator $\left[e, e^{\prime}\right]$ with $e, e^{\prime} \in \operatorname{idem}(R)$. We have $(1-e)-e^{\prime} \in \mathrm{U}(R)$ (by (2.13)(1)), so Theorem 2.8 implies that $(1-e)+e^{\prime} \in \mathrm{U}(R)$; that is, $1-v \in \mathrm{U}(R)$ for $v:=e-e^{\prime} \in \mathrm{U}(R)$ as in the last paragraph. On the other hand, $1+v=\left(1-e^{\prime}\right)+e \in \mathrm{U}(R)$ too, again by Theorem 2.8 since $\left(1-e^{\prime}\right)-e \in \mathrm{U}(R)$. This proves the "only if" part of (2). For the converse, assume that there is a unit $v=e-e^{\prime}$ for suitable $e, e^{\prime} \in \operatorname{idem}(R)$ such that $1 \pm v \in \mathrm{U}(R)$ for both signs. By Theorem 2.13(1), we will have Property $\mathrm{K}$ on $R$ if we can show that $1-e-e^{\prime} \in \mathrm{U}(R)$. Appealing to Theorem 2.8 once more (with the idempotent $e$ there replaced by $1-e)$, this would follow if $(1-e)+e^{\prime} \in \mathrm{U}(R)$ and $e+\left(1-e^{\prime}\right) \in \mathrm{U}(R)$. The former amounts to $1-v \in \mathrm{U}(R)$, while the latter amounts to $1+v \in \mathrm{U}(R)$. Since both of these conditions were given, we are done.

Our next goal is to study the possible presence of Property $\overline{\mathrm{K}}$ on $n \times n$ matrix rings over certain types of rings $T$. The first case we can treat without too much difficulty is when $(T, \mathfrak{m})$ is a local ring. Here, a judicious use of Corollary 2.16 enables us to (essentially) "replace" $T$ by the division $\operatorname{ring} T / \mathfrak{m}$. As we have pointed out before in (3.9), the condition $1_{T} \in \mathrm{U}(T)+\mathrm{U}(T)$ in the statement (2) below can be more simply expressed by $|T / \mathfrak{m}|>2$. However, we still prefer to use the former condition because of its more general nature. Similarly, in the statement (3) below, we prefer the condition "2 $\in \mathrm{U}(T)$ " to the equivalent condition "char $(T / \mathfrak{m}) \neq 2$ ".

Theorem 4.5. Let $(T, \mathfrak{m})$ be a local ring. Then $R=\mathbb{M}_{n}(T)$ has Property $\overline{\mathrm{K}}$ iff we are in one of the following cases:

(1) $n \in\{4,6,8, \ldots\}$.

(2) $n=2$ and $1_{T} \in \mathrm{U}(T)+\mathrm{U}(T)$.

(3) $n$ is odd and $2 \in \mathrm{U}(T)$.

Proof. If (1) or (2) holds, we know from Corollary 3.5 and Theorem 3.3 respectively that $R$ has Property $\mathrm{K}$, so of course $R$ has Property $\overline{\mathrm{K}}$. If (3) holds instead, then $<I_{n}, I_{n}>=2 I_{n} \in \mathrm{U}(R)$ shows that $R$ has Property $\overline{\mathrm{K}}$. Conversely, assume in the following that $R$ has Property $\overline{\mathrm{K}}$. If $n \in\{4,6,8, \ldots\}$, then (1) holds, so for the rest of the proof, we need only work with odd $n$ and $n=2$.

Case A. $T$ is a division ring. Fix an anti-commutator $\left\langle e, e^{\prime}\right\rangle \in \mathrm{U}(R)$ with (necessarily nonzero) $e, e^{\prime} \in \operatorname{idem}(R)$. First assume that $n=2$. If $e=I_{2},\left\langle e, e^{\prime}\right\rangle=2 e^{\prime} \in \mathrm{U}(R)$ implies that $2 \in \mathrm{U}(T)$, in which case $1_{T}=2^{-1}+2^{-1} \in \mathrm{U}(T)+\mathrm{U}(T)$. If $e \neq I_{2}$, we may assume (after a conjugation) that $e^{\prime}=E_{11}$. Writing $e=\left(\begin{array}{ll}a & b \\ c & d\end{array}\right)$, we have $\left\langle e, e^{\prime}\right\rangle=\left(\begin{array}{cc}2 a & b \\ c & 0\end{array}\right) \in \mathrm{U}(R)$, so $b, c \in \mathrm{U}(T)$. As $e^{2}=e$ implies that $a^{2}+b c=a$, we see that $a, 1-a$ are in $\mathrm{U}(T)$ too, with sum 1, so (2) holds. Finally, suppose $n$ is odd. Here we may assume that $e^{\prime}=\operatorname{diag}\left(I_{k}, 0_{n-k}\right)$ where $1 \leq k \leq n$. If $2 \notin \mathrm{U}(T)$, then $2=0 \in T$, and so $k<n$ (for otherwise $\left\langle e, e^{\prime}\right\rangle=2 e^{\prime}=0$, which is impossible). Writing $e=\left(\begin{array}{ll}A & B \\ C & D\end{array}\right)$ with $A \in \mathbb{M}_{k}(T)$ and $D \in \mathbb{M}_{n-k}(T)$, we have $\left\langle e, e^{\prime}\right\rangle=\left(\begin{array}{cc}0 & B \\ C & 0\end{array}\right)$. As $B$ and $C$ are non-square matrices, we can take a nonzero vector $v$ such that either 
$B v=0$ or $C v=0$. Then we'll have either $\left\langle e, e^{\prime}\right\rangle\left(\begin{array}{l}0 \\ v\end{array}\right)=0$ or $\left\langle e, e^{\prime}\right\rangle\left(\begin{array}{l}v \\ 0\end{array}\right)=0$, in contradiction to the fact that $\left\langle e, e^{\prime}\right\rangle \in \mathrm{U}(R)$. This completes the proof that $2 \in \mathrm{U}(T)$ when $n$ is odd.

Case B. $(T, \mathfrak{m})$ is a local ring. Let $\bar{T}$ be the division $\operatorname{ring} T / \mathfrak{m}$. By [La 1 : p. 57], $\operatorname{rad}\left(\mathbb{M}_{n}(T)\right)=\mathbb{M}_{n}(\operatorname{rad}(T))=\mathbb{M}_{n}(\mathfrak{m})$. Therefore,

$$
\mathbb{M}_{n}(T) / \operatorname{rad}\left(\mathbb{M}_{n}(T)\right)=\mathbb{M}_{n}(T) / \mathbb{M}_{n}(\mathfrak{m}) \cong \mathbb{M}_{n}(\bar{T})
$$

Since $\mathbb{M}_{n}(T)$ is an exchange ring (by [Wa] or [Ni]), Corollary 2.16 implies that it has Property $\overline{\mathrm{K}}$ iff $\mathbb{M}_{n}(\bar{T})$ does. Therefore, we are free to replace $T$ by $\bar{T}$ to assume that $T$ is a division ring, in which case we are fully covered by Case A above.

In general, we do not know exactly when a general matrix ring $\mathbb{M}_{n}(T)$ will have Property $\overline{\mathrm{K}}$ if $n$ is odd or $n=2$. Aside from the case where $T$ is local (as treated above in Theorem 4.5), a second manageable case is where $T$ is a commutative ring. Working under this assumption, it turns out that the more substantial case to consider is $n=2$. In this case, we were pleasantly surprised to find that Property $\mathrm{K}$ and Property $\overline{\mathrm{K}}$ are equivalent on $\mathbb{M}_{2}(T)$ ! To prove this, we start by first working out some easy facts about determinants of $2 \times 2$ matrices over a commutative ring.

Proposition 4.7. For $A, B \in R=\mathbb{M}_{2}(T)$ where $T$ is a commutative ring, we have

$$
\operatorname{det}(A+B)+\operatorname{det}(A-B)=2[\operatorname{det}(A)+\operatorname{det}(B)] \in T .
$$

Proof. Writing $A=\left(\begin{array}{ll}p & q \\ r & s\end{array}\right)$ and $B=\left(\begin{array}{ll}w & x \\ y & z\end{array}\right)$, a quick computation shows that

$$
\begin{gathered}
\operatorname{det}(A+B)=\operatorname{det}(A)+\operatorname{det}(B)+(p z+s w-q y-r x), \text { and } \\
\operatorname{det}(A-B)=\operatorname{det}(A)+\operatorname{det}(B)-(p z+s w-q y-r x) .
\end{gathered}
$$

Adding these two formulas gives the desired equation (4.8).

Corollary 4.9. In the notations of Proposition 4.7, if $2[\operatorname{det}(A)+\operatorname{det}(B)] \in \operatorname{rad}(T)$, then $A+B \in \mathrm{U}(R)$ iff $A-B \in \mathrm{U}(R)$. In particular, this "iff" statement holds in case $\operatorname{det}(A)+\operatorname{det}(B)=0$; or more specifically, if $\operatorname{det}(A)=\operatorname{det}(B)=0$.

Proof. The first "iff" statement holds on account of (4.8) since a matrix $C \in R$ is invertible iff $\operatorname{det}(C) \in \mathrm{U}(T)$, while for any $t, t^{\prime} \in T, t+t^{\prime} \in \operatorname{rad}(T)$ implies that $t \in \mathrm{U}(T)$ iff $t^{\prime} \in \mathrm{U}(T)$. The rest of the Corollary is clear.

With the above Corollary providing a crucial link between the invertibility of $A+B$ and $A-B$, we are now ready to prove the following result.

Theorem 4.10. Let $R=\mathbb{M}_{2}(T)$ where $T$ is a commutative ring. Then $R$ has Property $\mathrm{K}$ iff it has Property $\overline{\mathrm{K}}$.

Proof. Of course, only the "if" part is at stake. Before beginning its proof, we first point out that the "if" part does not mean that for any $e, e^{\prime} \in R,\left\langle e, e^{\prime}\right\rangle \in \mathrm{U}(R)$ 
implies that $\left[e, e^{\prime}\right] \in \mathrm{U}(R)$. Indeed, in the case where $2 \in \mathrm{U}(T)$, choosing $e=e^{\prime}=I_{2}$ gives $\left\langle e, e^{\prime}\right\rangle=2 I_{2} \in \mathrm{U}(R)$, but $\left[e, e^{\prime}\right]=0 \notin \mathrm{U}(R)$ if $T \neq 0$. (Nevertheless, under the assumption that $2 \in \mathrm{U}(T), R$ does have Property $\mathrm{K}$ by taking $a=b=2^{-1}$ in the proof of Theorem 3.1.) In the following, we assume that $R$ has Property $\overline{\mathrm{K}}$. We'll show that $R$ has Property $\mathrm{K}$ in two steps.

Step 1. The desired conclusion is true if $T$ is a connected ring; that is, if idem $(T)=$ $\{0,1\}$. Indeed, taking an invertible $\left\langle e, e^{\prime}\right\rangle$ (for suitable $e, e^{\prime} \in \operatorname{idem}(R)$ ), $\operatorname{det}(e)$ is either 1 or 0 . In the former case, $e$ is invertible, so $e=I_{2}$. Here, $\left\langle e, e^{\prime}\right\rangle=2 e^{\prime} \in \mathrm{U}(R)$ implies that $2 \in \mathrm{U}(T)$, so of course $R$ has Property K. We may thus assume that $\operatorname{det}(e)=0$, in which case $\operatorname{det}\left(e e^{\prime}\right)=\operatorname{det}\left(e^{\prime} e\right)=0$. As $e e^{\prime}+e^{\prime} e \in \mathrm{U}(R)$, the last part of Corollary 4.9 implies that $e e^{\prime}-e^{\prime} e \in \mathrm{U}(R)$, so $R$ has Property K.

Step 2. Assume that $R$ does not have Property K. By applying Zorn's Lemma to the family $\mathcal{F}$ of ideals $J_{i} \subseteq T$ such that $\mathbb{M}_{2}\left(T / J_{i}\right)$ does not have Property $\mathrm{K}$, we see that $\mathcal{F}$ has a maximal member (with respect to inclusion), say $J$. The maximal choice of $J$ implies that $T / J$ is a connected ring, for otherwise $\mathbb{M}_{2}(T / J)$ would have been a direct product of a pair of $2 \times 2$ matrix rings each of which has Property $\mathrm{K}$, in contradiction to the fact that $J \in \mathcal{F}$. On the other hand, $\mathbb{M}_{2}(T / J)$ is isomorphic to a factor ring of $\mathbb{M}_{2}(T)$, so it has Property $\overline{\mathrm{K}}$. As $T / J$ is a connected ring, what we have done in Step 1 implies that $\mathbb{M}_{2}(T / J)$ has Property K. This is a contradiction.

With the help of Theorem 4.10, we can now decide exactly when a matrix ring $\mathbb{M}_{n}(T)$ over a commutative ring $T$ will have Property $\bar{K}$. It is of interest to note that the conclusions in the following theorem happen to be identical to those of Theorem 4.5 over a (possibly noncommutative) local ring $T$, although the proofs are rather different in the key case where $n=2$.

Theorem 4.11. For any commutative ring $T, R=\mathbb{M}_{n}(T)$ has Property $\overline{\mathrm{K}}$ iff we are in one of the following cases:

(1) $n \in\{4,6,8, \ldots\}$.

(2) $n=2$ and $1_{T} \in \mathrm{U}(T)+\mathrm{U}(T)$.

(3) $n$ is odd and $2 \in \mathrm{U}(T)$.

Proof. If (1) or (2) holds, $R$ would have Property K, and therefore Property $\overline{\mathrm{K}}$. If (3) holds, of course $R$ has trivially Property $\overline{\mathrm{K}}$. Conversely, assume $R$ has Property $\overline{\mathrm{K}}$. If $n \in\{4,6,8, \ldots\}$, we are in Case (1). If $n=2$, we know from Theorem 4.10 that $R$ has Property K, so Theorem 3.14 implies that $1_{T} \in \mathrm{U}(T)+\mathrm{U}(T)$. Finally, let $n$ be odd. If $2 \notin \mathrm{U}(T)$, then 2 lies in some maximal ideal $\mathfrak{m} \subseteq T$. Since $\mathbb{M}_{n}(T / \mathfrak{m})$ is isomorphic to a factor ring of $R$, it also has Property $\overline{\mathrm{K}}$. As $T / \mathfrak{m}$ is a field of characteristic $2, \mathbb{M}_{n}(T / \mathfrak{m})$ would also have Property K, which would contradict Theorem 3.14. Therefore, we must have $2 \in \mathrm{U}(T)$, so we are in Case (3).

The result above suggests that the case of $\mathbb{M}_{2}(T)$ for commutative rings $T$ is of special interest in the treatment of Property K (or equivalently, Property $\overline{\mathrm{K}}$ by Theorem 4.10). We'll now conclude this paper by giving some more characterizations for these properties by using commutators and anti-commutators whose second entries are 
diagonalizable matrices instead of idempotent matrices. Such a replacement is by no means automatic since in general these two classes of matrices are logically independent.

Theorem 4.12. For $R=\mathbb{M}_{2}(T)$ where $T$ is a commutative ring, the following statements are equivalent:

(1) $R$ has Property $\mathrm{K}$ (or equivalently, $1_{T} \in \mathrm{U}(T)+\mathrm{U}(T)$ ).

(2) $[e, \delta] \in \mathrm{U}(R)$ for some $e \in \operatorname{idem}(R)$ and some diagonalizable matrix $\delta \in R$.

(3) $\langle e, \delta\rangle \in \mathrm{U}(R)$ for some $e \in \operatorname{idem}(R)$ and some diagonalizable matrix $\delta \in R$.

In the case where $T$ is an exchange ring, these statements are also equivalent to:

(4) $T$ has no ideal of index 2.

Proof. First assume (1) holds; say $1=a+b$ for some $a, b \in \mathrm{U}(T)$. Taking $e=$ $\left(\begin{array}{ll}a & b \\ a & b\end{array}\right) \in \operatorname{idem}(R)$ and $\delta=\operatorname{diag}(1,0)$, we have $[e, \delta] \in\left(\begin{array}{cc}0 & b \\ -a & 0\end{array}\right) \in \mathrm{U}(R)$, and $\langle e, \delta\rangle \in$ $\left(\begin{array}{cc}2 a & b \\ a & 0\end{array}\right) \in \mathrm{U}(R)$, so (2) and (3) both hold. Next, $(2) \Rightarrow(1)$ follows from the calculation in the proof of Case (2) in Theorem 3.8, after a reduction to the case where $\delta$ is diagonal. Now we come to the harder implication $(3) \Rightarrow(1)$. Given $e, \delta$ as in (3), we may again assume that $\delta=\operatorname{diag}(s, t)$ for some $s, t \in T$. Letting $e=\left(\begin{array}{ll}a & b \\ c & d\end{array}\right)$, the determinant of $\langle e, \delta\rangle$ is easily computed to be

$$
4 a d s t-b c(s+t)^{2} \in \mathrm{U}(T) .
$$

Let $g=\operatorname{det}(e)=a d-b c$, which is an idempotent in $T$. We have projection maps from $T$ onto $g T$ and onto $(1-g) T$. Taken together, these define a natural ring isomorphism from $T$ onto $g T \times(1-g) T$. Therefore, our job is reduced to showing that 1 is a sum of two units in both $g T$ and $(1-g) T$. Under the two projections, $g$ projects to the identity and to zero respectively. Thus, it suffices to argue in the following two cases.

Case 1. $g=1$. In this case, $e=I_{2}$, so $\langle e, \delta\rangle=2 \delta \in \mathrm{U}(R)$ implies that $2 \in \mathrm{U}(R)$. Thus, $2 \in \mathrm{U}(T)$, and so $1=2^{-1}+2^{-1} \in \mathrm{U}(T)+\mathrm{U}(T)$.

Case 2. $g=0$. In this case, $a d=b c$, so (4.13) shows that $b c \in \mathrm{U}(T)$. But $e \in$ idem $(R)$ implies that $a^{2}+b c=a$, so $a, 1-a$ are units in $T$, with sum 1 .

Finally, $(1) \Rightarrow(4)$ is always true, since the existence of an ideal $I \subseteq T$ of index two would imply that $R$ has a factor ring isomorphic to $\mathbb{M}_{2}\left(\mathbb{F}_{2}\right)$, which does not have Property K. Conversely, assume that $T$ is an exchange ring satisfying (4). To prove (1), we assume instead that $1_{T} \notin \mathrm{U}(T)+\mathrm{U}(T)$. Consider the nonempty family $\mathcal{G}$ of ideals $I_{i} \subseteq T$ for which 1 is not a sum of two units in $T / I_{i}$. Applying Zorn's Lemma to the family $\mathcal{G}$, we see that $\mathcal{G}$ has a maximal member $I$ (with respect to inclusion). The maximal choice of $I$ implies (as in the proof of (4.10)) that the commutative factor ring $T / I$ is connected. Since $T / I$ remains to be an exchange ring (and $T / I \neq 0$ ), it must be a local ring (by [Wa: Proposition 1]). As 1 is not a sum of two units in $T / I$, the local ring $T / I$ must have residue field $\mathbb{F}_{2}$. This implies that $T$ has a factor ring isomorphic to $\mathbb{F}_{2}$, which contradicts (4). 


\section{$\S 5$. Commutator Characterizations for $2 \times 2$ Matrix Rings}

According to Theorem 3.3, rings with Property K are precisely $2 \times 2$ matrix rings over base rings with a specific unit property. One may naturally ask: how about characterizations of the most general $2 \times 2$ matrix rings? In the literature, there is a simple criterion for these $((\mathrm{A}) \Leftrightarrow(\mathrm{H})$ in Theorem 5.1), that is due to Fuchs, Maxson and Pilz [FMP: (III.2)]. To make our exposition more useful and yet completely self-contained, we'll reprove the Fuchs-Maxson-Pilz theorem in a much expanded form below, following the idea of using anti-commutators (as well as commutators) in [La 4 : p. 349] but not assuming any general matrix ring recognition theorems in [La: $\S 17]$. In the following result, the statements $(\mathrm{B})$ through $(\mathrm{G})$ are expressed in terms of anti-commutators, while $(\mathrm{I}),(\mathrm{J})$ and $(\mathrm{K})$ are expressed in terms of commutators.

Theorem 5.1. For any ring $R$, the following statements are equivalent:

(A) $R \cong \mathbb{M}_{2}(S)$ for some ring $S$.

(B) There exist $p, q \in R$ with $p^{2}=q^{2}=0$ such that $\langle p, q\rangle=1$.

(C) There exist $p, q \in R$ with $p^{2}=q^{2}=0$ such that $\langle p, q\rangle \in \mathrm{U}(R)$.

(D) There exist $v \in \mathrm{U}(R)$ and $p \in R$ with $p^{2}=0$ such that $\langle p, v\rangle=1$.

(E) There exist $v \in \mathrm{U}(R)$ and $p \in R$ with $p^{2}=0$ such that $\langle p, v\rangle \in \mathrm{U}(R)$.

(F) There exist $r, p \in R$ with $p^{2}=0$ such that $\langle p, r\rangle=1$.

(G) There exist $r, p \in R$ with $p^{2}=0$ such that $\langle p, r\rangle \in \mathrm{U}(R)$.

(H) There exist $p, q \in R$ with $p^{2}=q^{2}=0$ and $p+q \in \mathrm{U}(R)$.

(I) There exist $p, q \in R$ with $p^{2}=q^{2}=0$ such that $[p, q] \in \mathrm{U}(R)$.

(J) There exist $v \in \mathrm{U}(R)$ and $p \in R$ with $p^{2}=0$ such that $[p, v] \in \mathrm{U}(R)$.

$(\mathrm{K})$ There exist $r, p \in R$ with $p^{2}=0$ such that $[p, r] \in \mathrm{U}(R)$.

Proof. (A) $\Rightarrow(\mathrm{B})$. If (say) $R=\mathbb{M}_{2}(S)$, then (B) holds for $p=E_{12}$ and $q=E_{21}$.

$(\mathrm{B}) \Rightarrow(\mathrm{C})$ is a tautology.

$(\mathrm{C}) \Rightarrow(\mathrm{H})$. For $p, q$ as in $(\mathrm{C})$, we have $(p+q)^{2}=\langle p, q\rangle \in \mathrm{U}(R)$, so $p+q \in \mathrm{U}(R)$.

$(\mathrm{H}) \Rightarrow(\mathrm{D})$. For $p, q \in R$ as in $(\mathrm{H})$, let $v$ be the inverse of $u:=p+q \in \mathrm{U}(R)$. Left multiplying by $q$ and right multiplying by $p$ give $q u=q p$ and $u p=q p$ respectively. Equating these gives $v q=p v$, so $\langle p, v\rangle=p v+v p=v q+v p=v u=1$.

$(\mathrm{D}) \Rightarrow(\mathrm{G})$ is a tautology.

$(\mathrm{G}) \Rightarrow(\mathrm{F})$. For $r, p$ as in $(\mathrm{G})$, let $w=p r+r p \in \mathrm{U}(R)$. Left multiplying by $p$ gives $p w=p r p$, and right multiplying by $p$ gives $w p=p r p$. Thus, $p w=w p$, so left multiplying by $w^{-1}$ yields $1=w^{-1} p r+w^{-1} r p=p\left(w^{-1} r\right)+\left(w^{-1} r\right) p$, which verifies (F).

$(\mathrm{F}) \Rightarrow(\mathrm{A})$. For $r, p$ as in $(\mathrm{F})$, left multiplying $p r+r p=1$ by $e:=r p$ shows that $e \in \operatorname{idem}(R)$, with complementary idempotent $f:=p r$. By [Laa $:(21.20)], e, f$ are isomorphic idempotents. Thus, we have $R_{R}=e R \oplus f R \cong e R \oplus e R$ as right $R$-modules. Taking endomorphism rings gives $R \cong \operatorname{End}_{R}(e R) \cong \mathbb{M}_{2}(e R e)$.

$(\mathrm{D}) \Leftrightarrow(\mathrm{E})$. We need only show that $(\mathrm{E}) \Rightarrow(\mathrm{D})$, so let $w:=p v+v p \in \mathrm{U}(R)$ where $p^{2}=0$ and $v \in \mathrm{U}(R)$. Applying the same proof for $(\mathrm{G}) \Rightarrow(\mathrm{F})$ here gives $1=$ $p\left(w^{-1} v\right)+\left(w^{-1} v\right) p$. Since $w^{-1} v \in \mathrm{U}(R)$, this proves (D). 
$(\mathrm{A}) \Rightarrow\{(\mathrm{I}),(\mathrm{J})$ and $(\mathrm{K})\}$. If (say) $R=\mathbb{M}_{2}(S)$, then (I) holds for $p=E_{12}$ and $q=E_{21}$, and $(\mathrm{J}),(\mathrm{K})$ both hold for $p=E_{12}$ and $v=r=E_{12}+E_{21}$.

$(\mathrm{I}) \Rightarrow(\mathrm{C})$. Given $p, q$ in $(\mathrm{I})$, let $w:=[p, q] \in \mathrm{U}(R)$. Repeating the first steps in the proof of $(\mathrm{G}) \Rightarrow(\mathrm{F})$, we can show here that $w$ anti-commutes with both $p$ and $q$. Thus,

$$
1=w^{-1} p q-w^{-1} q p=-p\left(w^{-1} q\right)-\left(w^{-1} q\right) p=-\left\langle p, w^{-1} q\right\rangle .
$$

Since $\left(w^{-1} q\right)^{2}=w^{-1} q w^{-1} q=-w^{-2} q^{2}=0$, this verifies $(\mathrm{C})$.

$(\mathrm{J}) \Rightarrow(\mathrm{K}) \Rightarrow(\mathrm{G})$. It suffices to prove the latter implication. Given $w:=p r-r p \in \mathrm{U}(R)$ as in $(\mathrm{K})$, we have again $w p=-p w$. Thus, by repeating the calculations in (5.2) (with $q$ replacing $r$ ), we get $1=-\left\langle p, w^{-1} r\right\rangle$. Since $p^{2}=0$, this verifies $(\mathrm{G})$.

Remark 5.3. It would be tempting to think that in the statements (B), (D) and (F), we can replace the anti-commutators by commutators and get three more equivalent statements in terms of commutators. However, this is not the case! In fact, given (A), we may not be able to get an equation $[p, q]=1$ with $p^{2}=q^{2}=0$. Indeed, if $R=\mathbb{M}_{2}(S)$ for a commutative ring $S$, any commutator in $R$ will have zero trace, while the identity matrix $I_{2}$ has trace 2. Similarly, we must refrain from trying to change the anti-commutator into a commutator in the statements (D) and (F). As for statement $(\mathrm{H})$ (the Fuchs-Maxson-Pilz characterization for (A)), it is easy to see that we also cannot change the requirement $p+q \in \mathrm{U}(R)$ into $p+q=1 \in R$ (or $p+q$ being a central unit), since this would have implied that $p=q=0$, and hence $R=0$ !

Remark 5.4. As we have pointed out in the paragraph preceding Theorem 5.1, the proof of that theorem was designed to be entirely self-contained so that it can be read independently of the standard recognition theorems for matrix rings as developed, for instance, in $\left[\mathrm{La}_{3}\right]$. In the simplest case, according to $\left[\mathrm{La}_{3}:(17.10)\right]$, a standard characterization for a ring $R$ to be a $2 \times 2$ matrix ring is that there exist $p, r, r^{\prime} \in R$ with $p^{2}=0$ such that $p r+r^{\prime} p=1$. This condition can thus be added to all the other equivalent conditions listed in Theorem 5.1. We note that it is ostensibly "weaker" (as a sufficient condition for (A)) than the three conditions (B), (D), and (F).

At this point, it would be helpful to recall a key definition introduced in our earlier work $[\mathrm{KL}]$ on invertible commutators. In that paper, we defined a ring element $a \in R$ to be completable if there exists $r \in R$ such that $[a, r] \in \mathrm{U}(R)$. Using this terminology, the equivalence of $(\mathrm{A})$ and $(\mathrm{K})$ in Theorem 5.1 says precisely that $R$ is a $2 \times 2$ matrix ring (over some other ring) iff $R$ has a square-zero completable element. Inspired by this fact (as well as by our earlier Theorem 3.3), we stumbled upon the idea of looking for completable idempotents in $R$. It was a pleasant surprise to us that the existence of a completable idempotent in a ring $R$ turns out to be also equivalent to $R$ being a $2 \times 2$ matrix ring. This fact and some of its variations are collected in the new characterization theorem below for $2 \times 2$ matrix rings. Recall from Corollary 3.4 that, in the special case where $2 \in \mathrm{U}(R)$, we did know that $R$ is a $2 \times 2$ matrix ring iff $R$ has Property $\mathrm{K}$ (that is, $\left[e, e^{\prime}\right] \in \mathrm{U}(R)$ for some $\left.e, e^{\prime} \in \operatorname{idem}(R)\right)$. However, the following new equivalence result, like Theorem 5.1, is valid for all rings.

Theorem 5.5. For any ring $R$, the following statements are equivalent: 
(1) $R \cong \mathbb{M}_{2}(S)$ for some ring $S$.

(2) There exist $e \in \operatorname{idem}(R)$ and $r \in R$ with $r^{2}=1$ such that $[e, r] \in \mathrm{U}(R)$.

(3) There exist $e \in \operatorname{idem}(R)$ and $r \in R$ with $r^{2}=-1$ such that $[e, r] \in \mathrm{U}(R)$.

(4) There exist $e \in \operatorname{idem}(R)$ and $r \in R$ with $r^{3}=1$ such that $[e, r] \in \mathrm{U}(R)$.

(5) There exist $e \in \operatorname{idem}(R)$ and $r \in \mathrm{U}(R)$ such that $[e, r] \in \mathrm{U}(R)$.

(6) $R$ has a completable idempotent.

(7) There exist $e \in \operatorname{idem}(R)$ and $r \in R$ with $r^{2}=0$ such that $[e, r] \in \mathrm{U}(R)$.

(8) There exist $e \in \operatorname{idem}(R)$ and a nilpotent $r \in R$ such that $[e, r] \in \mathrm{U}(R)$.

Proof. We first prove that (1) implies (2), (3), (4) and (7). Indeed, if $R=\mathbb{M}_{2}(S)$ for some ring $S$, we can take $e=\left(\begin{array}{ll}1 & 0 \\ 0 & 0\end{array}\right), r_{2}=\left(\begin{array}{ll}0 & 1 \\ 1 & 0\end{array}\right), r_{3}=\left(\begin{array}{cc}0 & 1 \\ -1 & 0\end{array}\right), r_{4}=\left(\begin{array}{cc}-1 & 1 \\ -1 & 0\end{array}\right)$, and $r_{7}=\left(\begin{array}{cc}1 & 1 \\ -1 & -1\end{array}\right)$. Then $e^{2}=e, r_{2}^{2}=r_{4}^{3}=1, r_{3}^{2}=-1$, and $r_{7}^{2}=0$, and we check easily that $\left[e, r_{i}\right] \in \mathrm{U}(R)$ for all $i$.

$(7) \Rightarrow(8) \Rightarrow(6)$ are tautologies. [We note on the side that $(7)$ also trivially implies $(\mathrm{K})$ (and hence $(\mathrm{A})$ ) in Theorem 5.1, although this information is not needed here.]

$(6) \Rightarrow(1)$. Let $e=e^{2} \in R$ and $r \in R$ be such that the following commutator is a unit:

$$
[e, r]=e r-r e=e r(1-e)-(1-e) r e .
$$

Letting $p=e r(1-e)$ and $q=-(1-e) r e$, we have clearly $p^{2}=q^{2}=0$, and $p+q \in \mathrm{U}(R)$ according to (5.6). Thus, $(\mathrm{H}) \Rightarrow(\mathrm{A})$ in Theorem 5.1 gives (1).

The proof is now complete, since $\{(2)$ or $(3)$ or $(4)\} \Rightarrow(5) \Rightarrow(6)$ are tautologies.

It is of interest to point out that, in the various criteria above, $r$ can be chosen to be a unit (respectively, a square root of \pm 1 or a cubic root of 1 ), or a nilpotent element (respectively, a square-zero element), but not necessarily an idempotent. We note also that, in the criterion (5) above, the commutator $[e, r]$ cannot be replaced by the anti-commutator $\langle e, r\rangle$. Indeed, if we take $R$ to be any nonzero commutative ring with $2 \in \mathrm{U}(R)$, and choose $e=r=1$, then $\langle e, r\rangle \in \mathrm{U}(R)$. Here, $R$ cannot possibly be a $2 \times 2$ matrix ring. Nevertheless, we do have the following result for anti-commutators.

Corollary 5.7. If $R$ is a ring with an idempotent $e$ and an element $r$ such that $\langle e, r\rangle \in \mathrm{U}(R)$, then $R / 2 R \cong \mathbb{M}_{2}(S)$ for some ring $S$.

Proof. In $\bar{R}=R / 2 R$, we have $\overline{e r-r e}=\overline{e r+r e} \in \mathrm{U}(\bar{R})$, so we can apply $(5) \Rightarrow(1)$ in Theorem 5.5 to the factor ring $\bar{R}$.

Emboldened by the fact that $2 \times 2$ matrix rings are characterized by the existence of completable idempotents as well as the existence of completable square-zero elements, we were led to the consideration of completable involutions as well. (By an involution, we simply mean an element $u \in R$ with $u^{2}=1$.) In ring theory, it is rather rare that idempotents, involutions and square-zero elements would play parallel roles in the treatment of a certain problem or property. But for the problem of characterizing $2 \times 2$ matrix rings, this does turn out to be the case, as the following result shows. 
Theorem 5.8. A ring $R$ is a $2 \times 2$ matrix ring iff it has a completable involution.

Proof. If $R=\mathbb{M}_{2}(S)$ for some ring $S$, we have already pointed out earlier that the involution $E_{12}+E_{21} \in R$ is completable. Conversely, assume that there exist $u, r \in R$ with $u^{2}=1$ such that $v:=[u, r] \in \mathrm{U}(R)$. Repeating the idea (in the proof of (5.1)) of left and right multiplying by $u$, and keeping in mind the equation $u^{2}=1$, we see here that $u v=-v u$. Thus,

$$
1=v^{-1} u r-v^{-1} r u=(1-u) v^{-1} r-v^{-1} r(1+u) ;
$$

that is, $1=e+f$, where $e:=(1-u) v^{-1} r$ and $f:=-v^{-1} r(1+u)$. Since $f e=0$, we have $f \in \operatorname{idem}(R)$, with complementary idempotent $e$. It suffices to show that $e$ and $f$ are isomorphic idempotents, as that will show (as in $(\mathrm{F}) \Rightarrow(\mathrm{A})$ in the proof of Theorem 5.1) that $R \cong \mathbb{M}_{2}(e R e)$. Right multiplying (5.9) by $1-u$ and left multiplying it by $1+u$ give the following two "von Neumann regularity" equations:

$$
(1-u) v^{-1} r(1-u)=1-u \text {, and }(1+u)\left(-v^{-1} r\right)(1+u)=1+u .
$$

From the former, we have $e R=(1-u) R$. From the latter, we have $R f=R(1+u)$, which is well known to imply that $f R \cong(1+u) R$ as right $R$-modules. (For a full proof of this implication, see $\left[\mathrm{La}_{2}\right.$ : Exercise 1.17].) Noting that

$$
(1-u) R=(1-u) v R=v(1+u) R \cong(1+u) R,
$$

we conclude that $e R \cong f R$, as desired.

Remark 5.10. In view Theorem 5.8 (and parts of Theorem 5.5), it might be tempting to surmise that the existence of a completable element $u \in R$ with $u^{n}=1$ for some $n \geq 3$ might also imply that $R$ is a $2 \times 2$ matrix ring (over some other ring). However, this is not the case. For instance, let $R$ be Hamilton's quaternion division algebra generated over $\mathbb{R}$ by $i, j$, with the relations $i^{2}=j^{2}=-1$ and $i j=-j i$. Let $u=$ $a+b i(a, b \in \mathbb{R})$ be a primitive $n$-th of unity in $\mathbb{R}[i] \cong \mathbb{C}$. If $n \geq 3$, then $b \neq 0$, and $[u, j]=[a+b i, j]=b[i, j]=2 b i j \in \mathrm{U}(R)$. This shows that $u \in R$ is completable. However, being a division algebra, $R$ is not a $2 \times 2$ matrix ring over any ring. In a similar vein, if a ring $R$ has a completable element $w$ such that $w^{n} \in\{0, w\}$ for some $n \geq 3, R$ need not be a $2 \times 2$ matrix ring either. For instance, let $R=\mathbb{M}_{3}(S)$ over a ring $S$. For the two matrices

$$
w=\left(\begin{array}{ccc}
1 & 1 & 0 \\
0 & 0 & 1 \\
0 & 0 & -1
\end{array}\right) \text { and } w_{1}=\left(\begin{array}{ccc}
1 & 0 & 0 \\
0 & 0 & 0 \\
1 & 1 & 0
\end{array}\right) \text {, we have }\left[w, w_{1}\right]=\left(\begin{array}{ccc}
0 & -1 & 0 \\
1 & 1 & 0 \\
-2 & -2 & -1
\end{array}\right) \in \mathrm{U}(R) .
$$

Thus, $w$ is completable in $R$, and we can check easily that $w^{3}=w$. However, if we choose $S$ to be a division ring (or $S=\mathbb{Z}$ ), $R$ is not a $2 \times 2$ matrix ring over any ring. The case where $w^{n}=0$ for some $n \geq 3$ can be handled similarly by taking instead $w=\left(\begin{array}{lll}0 & 1 & 0 \\ 0 & 0 & 1 \\ 0 & 0 & 0\end{array}\right)$ and $w_{2}=\left(\begin{array}{ccc}0 & 0 & 0 \\ 1 & 0 & 1 \\ 1 & 0 & 0\end{array}\right)$, with $w^{3}=0$ and $\left[w, w_{2}\right]=\left(\begin{array}{ccc}1 & 0 & 1 \\ 1 & -1 & 0 \\ 0 & -1 & 0\end{array}\right) \in \mathrm{U}(R)$.

Acknowledgments. We are grateful to Professor S. K. Berberian who kindly communicated to us his detailed personal notes on Kaplansky's "Exercise 6" from [Ka: p. 25]. 
These notes helped us come to a better understanding of Kaplansky's proposed solution of his exercise, which eventually led to the present work. We thank Professor G. M. Bergman for his valuable contribution $\left[\mathrm{Be}_{2}\right]$ toward the validation of Example 3.6, and we also thank the referee of this paper for his/her many thoughtful comments.

\section{References}

$\left[\mathrm{Be}_{1}\right]$ G. M. Bergman: Modules over coproducts of rings. Trans. Amer. Math. Soc. 200 (1974), 1-32.

$\left[\mathrm{Be}_{2}\right]$ G. M. Bergman: Email communication, July 3, 2017.

[BD] R. Bott and R. J. Duffin: On the algebra of networks. Trans. Amer. Math. Soc. 74 (1953), 99-109.

$\left[\mathrm{Co}_{1}\right]$ P.M. Cohn: Some remarks on the invariant basis property. Topology 5 (1966), 215-228.

$\left[\mathrm{CO}_{2}\right]$ P.M. Cohn: Some remarks on projective-free rings. Algebra Universalis 49 (2003), 159-164.

$\left[\mathrm{Co}_{3}\right]$ P.M. Cohn: Another criterion for a ring to be projective-free. Bull. London Math. Soc. 37 (2005), 857-859.

[FMP] P. R. Fuchs, C. J. Maxson and G. F. Pilz: On rings for which homogeneous maps are linear. Proc. Amer. Math. Soc. 112 (1991), 1-7.

[Go] K. R. Goodearl: Von Neumann Regular Rings. Second Edition, Robert E. Krieger Publishing Company, Malabar, Florida, 1991.

[GH] K. R. Goodearl and D. Handelman: Simple self-injective rings. Comm. Alg. 3 (1975), 797-834.

[GW] H. Grover, Z. Wang, D. Khurana, J. Chen and T. Y. Lam: Sums of units in rings. J. Alg. Appl. 13 (2014), 1350072, 10 pp.

[He] M. Henriksen: Two classes of rings generated by their units. J. Alg. 31 (1974), 182-193.

[Ka] I. Kaplansky: Rings of Operators. W. A. Benjamin, New York, 1968.

[Kt] T. Kato: Perturbation Theory for Linear Operators. Second Ed., Classics in Mathematics, Springer-Verlag, Berlin-Heidelberg-New York, 1995.

[KL] D. Khurana and T. Y. Lam: Invertible commutators in matrix rings. J. Algebra Appl. 10 (2011), 51-71.

[KLS] D. Khurana, T. Y. Lam and N. Shomron: A quantum-trace determinantal formula for matrix commutators, and applications. Lin. Alg. Appl. 436 (2012), 2380-2397. 
$\left[\mathrm{KR}_{1}\right]$ J. J. Koliha and V. Rakočević: Invertibility of the sum of idempotents. Lin. Multilin. Algebra 50 (2002), 285-292.

$\left[\mathrm{KR}_{2}\right]$ J. J. Koliha and V. Rakočević: Invertibility of the difference of idempotents. Lin. Multilin. Algebra 51 (2003), 97-110.

[La $]$ T. Y. Lam: A First Course in Noncommutative Rings. Second Edition, Graduate Texts in Math., Vol. 131, Springer-Verlag, Berlin-Heidelberg-New York, 2001.

[La $\mathrm{La}_{2}$ T. Y. Lam: Exercises in Classical Ring Theory. Second Edition, Problem Books in Mathematics, Springer-Verlag, Berlin-Heidelberg-New York, 2003.

[La $]$ T. Y. Lam: Lectures on Modules and Rings. Graduate Texts in Math., Vol. 189, Springer-Verlag, Berlin-Heidelberg-New York, 1999.

[La $\left.{ }_{4}\right]$ T.Y. Lam: Exercises in Modules and Rings. Problem Books in Mathematics, Springer-Verlag, Berlin-Heidelberg-New York, 2007.

[Le] W. G. Leavitt: Rings without invariant basis number. Proc. Amer. Math. Soc. 8 (1957), 322-328.

[Ni] W. K. Nicholson: Lifting idempotents and exchange rings. Trans. Amer. Math. Soc. 229 (1977), 269-278.

[Sa] E. M. de Sá: The rank of a difference of similar matrices. Portugal. Math. 46 (1989), 177-187.

[SG] G. Song and X. Guo: Diagonability of idempotent matrices over noncommutative rings. Lin. Multilin. Algebra 297 (1999), 1-7.

[Wa] R. B. Warfield: Exchange rings and decompositions of modules. Math. Ann. 199 (1972), 31-36.

Footnotes:

2010 AMS Subject Classification: 12E15, 15B33, 15B36, 16E50, 16N40, 16 U60.

Keywords: Units, idempotents, commutators, anti-commutators, Bott-Duffin inverse, matrix rings, simple artinian rings, exchange rings, commutative rings.

Department of Mathematics

Panjab University

Chandigarh 160 014, India

dkhurana@pu.ac.in

Department of Mathematics

University of California

Berkeley, CA 94720, USA

lam@math . berkeley . edu 